\title{
A comparison of cognitive performances between neuromyelitis optica spectrum disorder and multiple sclerosis patients in Indian context
}

Swati Kumar, Goutam Gangopadhyay, Atanu Biswas, Souvik Dubey º, Alak Pandit, Shambaditya Das and Biman Kanti Ray

\begin{abstract}
Background: To compare the frequency and pattern of cognitive impairment in neuromyelitis optica spectrum disorder (NMOSD) and multiple sclerosis (MS) patients.

Results: Twenty NMOSD and forty MS patients were included. Clinical and detailed neuropsychological assessment was done using frontal assessment battery and Kolkata cognitive battery supplemented with additional standard tests for different domains of cognitive functions. Domain wise tests were performed and compared. 15/20 (75\%) NMOSD and 32/40 (80\%) MS patients had cognitive impairment $(p=0.65)$. Executive function, verbal fluency, information processing speed, visuo-constructional ability, attention, complex calculation, and memory were more commonly involved in NMOSD in decreasing order. Compared to MS, the pattern was similar except that verbal fluency was more impaired in NMOSD. Expanded Disability Status scale (EDSS) correlated with cognitive involvement in NMOSD $(p=0.02)$ as against MS.

Conclusions: Executive function, verbal fluencies, and information processing speed were more affected compared to visual and verbal memory in NMOSD patients. The pattern of cognitive performance was similar in the MS group, even though clinical and radiological characteristics and pathophysiology is different, suggesting similar brain involvement.
\end{abstract}

Keywords: Neuromyelitis optica spectrum disorders, Multiple sclerosis, Cognitive impairment

\section{Background}

Neuromyelitis optica spectrum disorder (NMOSD) is an inflammatory and demyelinating condition of the central nervous system (CNS) characterized by optic neuritis and longitudinally extensive transverse myelitis. Currently, brain involvement in NMOSD is well recognized, and attacks on the brain may even occur as the first manifestation of NMOSD [1, 2]. NMOSD is caused by antibody (IgG)-mediated attack against Aquaporin 4

\footnotetext{
*Correspondence: drsouvik79@gmail.com

Department of Neurology, Bangur Institute Of Neurosciences, IPGMER and

SSKM Hospital, 52 1/A Sambhu Nath Pandit Road, Bhowanipore, Kolkata, West Bengal 700020, India
}

\section{SpringerOpen}

(AQP4), which is a bidirectional water channel protein on the foot process of astrocytes present all over CNS. This further sets up a cascade of reactions ultimately altering the synaptic plasticity of neurons. Cognitive impairment is considered to be an attack independent progressive symptom in NMOSD patients, presumably involving a similar phenomenon [3].

Cognitive impairment (CI) in NMOSD as seen in the contemporary studies is present in $30-70 \%$ of patients, predominantly involving alteration in attention, language, memory, processing speed, and executive function [3].
(๑) The Author(s). 2021 Open Access This article is licensed under a Creative Commons Attribution 4.0 International License, which permits use, sharing, adaptation, distribution and reproduction in any medium or format, as long as you give appropriate credit to the original author(s) and the source, provide a link to the Creative Commons licence, and indicate if changes were made. The images or other third party material in this article are included in the article's Creative Commons licence, unless indicated otherwise in a credit line to the material. If material is not included in the article's Creative Commons licence and your intended use is not permitted by statutory regulation or exceeds the permitted use, you will need to obtain permission directly from the copyright holder. To view a copy of this licence, visit http://creativecommons.org/licenses/by/4.0/. 
In multiple sclerosis (MS), which is also an inflammatory demyelinating disorder of CNS, CI has been extensively studied and found to be present in $45-65 \%$ of the patients during the course of the disease [4]. The cognitive dysfunction in MS is usually subtle early in the course of the disease, although dementia has been observed in $20-30 \%$ of the severely impaired patients. The pattern of involvement is predominantly subcortical. In general, the cognitive domains involved are complex attention, information processing speed, verbal and visuo-spatial memory, and executive function; whereas language, semantic memory, and attention span are rarely involved.

There have been only a few studies till now documenting CI in NMOSD patients. Although no significant differences in the frequency and pattern of cognitive performance have been reported between NMOSD and MS patients [5-7], these results should be interpreted with caution due to the small sample size and variation in the test cut-offs considered indicative of CI.

Thus, in this study, we have compared the cognitive profile of NMOSD and MS patients to see if and how they differ. Moreover, we have looked for any association between the demographic factors, clinical disability, and MRI lesion load with the cognitive performance in each group.

\section{Methods}

A total of 20 NMOSD and 40 MS patients were recruited in the Department of Neurology, Bangur Institute of Neurosciences, Kolkata for one and a half year (March 2018-October 2019) for a cross-sectional study. MS was diagnosed by Revised McDonald's Criteria 2010 [8], and NMOSD was diagnosed by International Panel for NMO Diagnosis (IPND) criteria 2015 [9]. All patients who fulfilled the criteria and gave proper consent were included in the study after the acute phase was treated (at least 1 month and afterwards were in remission). The exclusion criteria were acute relapses of MS or NMOSD, comorbid neurological diseases other than NMOSD or MS, a history of psychiatric illness including depression, and alcohol and substance abuse. None of our patients had severe visual or physical disability, particularly of the dominant hand so as to restrict their performance of the cognitive tests. The parameters studied were current age, gender, educational level, disease duration, number of relapses, and disability which was measured by the Expanded Disability Status Scale (EDSS) [10], Oligoclonal band (OCB) in cerebrospinal fluid (CSF), and AQP4 positivity in serum (by cell based assay). This study was approved by the institutional Ethical Committee. Informed consent was obtained from all patients.

\section{Neuropsychological assessment}

The assessment was performed by trained neurologist under the guidance of psychologist, using frontal assessment battery (FAB) [11] and Kolkata cognitive battery (KCB) [12] supplemented with additional standard tests for different domains of cognitive functions. Attention was assessed using digit span forward, digit span backward, "A vigil" test, serial subtraction (e.g., 100-7, 20-3) as scored in Montreal cognitive assessment (MOCA )[13] and extended version of KCB. Information processing speed (IPS) was assessed using timed 100-7, timed trail A and timed trail B tests. IPS depends on time. For every unique population, there must be different normal variance of timing. To overcome this limitation, here we made our own time frame for IPS including control population and compared it with our patients. Language was assessed under spontaneous speech; comprehension namely single word, simple comprehension (asking to hold the pen or paper), complex comprehension (asking to hold pen and then the paper), and syntactic comprehension (using passive voice sentences); and fluency was assessed under category fluency (telling as many animal names in $1 \mathrm{~min}$, fruit names in $1 \mathrm{~min}$ as possible, cut-off taken as 7), letter or lexical fluency asking to say as many words in $1 \mathrm{~min}$ as possible excluding proper nouns starting with a particular alphabet scoring as in FAB; repetition of single words, like eccentricity, Hippopotamus, unintelligible, statistician, used in patient's vernacular language scoring as in Addenbrooke's cognitive examination (ACE III) [14] and string of words, like no ifs, ands or buts, used in patient's vernacular language scoring as in MMSE; reading with or without comprehension seen by reading a passage and complex, silent, difficult words; writing assessed by asking to write a meaningful sentence; naming assessed by real object naming. Memory was assessed under verbal memory using word list memory task indicating immediate recall, delayed word list memory task, delayed recognition memory task, autobiographical and semantic memory, and scored as in KCB. Visual memory was assessed using brief visual memory test revised [15]. Visuoconstructional skill was assessed using $2 \mathrm{D}$ and $3 \mathrm{D}$ geometric figure drawing. Calculation was assessed as simple (single digit) and complex (double digits). Visuoperceptual ability was assessed using dot counting and fragmented letters and scored as in ACE III. Praxis was assessed by asking to pantomime verbal command, imitate the examiner, pantomime seeing picture of the tool, pantomime using the actual tool, showing a picture, and asking what instrument was needed to push the nail further, which object would you use to keep your teeth clean and how would you do so. Gnosis was tested by identification of pictures of famous personalities, tools and objects, and their functions. 


\section{Executive function}

Executive function was assessed using FAB, as well as individual subdomains as follows: abstraction was assessed using proverb interpretation and similarities; mental flexibility using trail making A and B (a shorter version as used in MOCA), go-no-go test; verbal fluency or lexical fluency as a part of FAB; judgement by asking reallife scenario questions; set shifting ability by Luria test ( pattern as well as motor palm fist edge test); and planning by clock face drawing test scored as in ACE III.

\section{Magnetic resonance imaging (MRI)}

Radiological parameters were assessed by MRI 3 Tesla using Siemens magnetom verio 3T machine. The sequences studied were T1 (TR < 500, TE 15-20), T2 (TR 4000, TE 100), (FLAIR) fluid attenuated inversion recovery ( TR 8000, TE 70-80, TI 2500), diffusion-weighted imaging (DWI), apparent diffusion coefficient (ADC) mapping, short TI inversion recovery (STIR), gradient echo (GRE) of the brain, orbits, and spinal cord wherever relevant with or without gadolinium contrast. The parameters studied were number of lesions grouped as $\leq$ $4,>4-\leq 9,>9$, lesion location, and contrast enhancement.

The cognitive performance was estimated in the form of FAB score and number of cognitive domains involved and number of executive subdomains involved. Its association with demographic parameters, such as age, sex, and formal education; and clinical parameters, such as disease duration, number of relapses, EDSS, OCB positivity (for MS), and MRI lesion load, was calculated.

\section{Statistical analysis}

The data has been analyzed using SPSS (version 25.0; SPSS Inc., Chicago, IL, USA) and GraphPad Prism version 5. Data have been summarized as mean and standard deviation for numerical variables and count and percentages for categorical variables.

Two-sample $t$ tests was used for a difference in mean involved independent samples or unpaired samples. Paired $t$ tests were a form of blocking and had greater power than unpaired tests.

One-way analysis of variance (one-way ANOVA) was a technique used to compare means of three or more samples for numerical data (using the F distribution).

A chi-squared test ( $\chi 2$ test) was any statistical hypothesis test wherein the sampling distribution of the test statistic is a chi-squared distribution when the null hypothesis is true. Without other qualification, chi-squared test often is used as short for Pearson's chi-squared test. Unpaired proportions were compared by chi-square test or Fischer's exact test, as appropriate.

Correlation was calculated by Pearson correlation analysis. The Pearson product-moment correlation coefficient was a measure of the linear dependence between two variables $\mathrm{X}$ and $\mathrm{Y}$. Explicit expressions that can be used to carry out various $t$ tests are given below. In each case, the formula for a test statistic that either exactly follows or closely approximates a $t$ distribution under the null hypothesis is given. Also, the appropriate degrees of freedom are given in each case. Each of these statistics can be used to carry out either a one-tailed test or a two-tailed test.

Once a $t$ value is determined, a $p$ value can be found using a table of values from Student's $t$ distribution. If the calculated $p$ value is below the threshold chosen for statistical significance (usually the 0.10 , the 0.05 , or 0.01 level), then the null hypothesis is rejected in favor of the alternative hypothesis. $p$ value $\leq 0.05$ was considered for statistically significant.

\section{Results}

The results have been summarized in Tables 1, 2, 3, 4, and 5.

There was no significant difference of mean age and gender ratio between two groups, although mean age MS patients and proportion of female subjects in NMOSD were higher. The MS patients had a higher education level $(p=0.005)$, a longer duration of disease $(p<0.0001)$, and a greater number of relapses $(p<$ $0.0001)$. Moreover, MS patients had a higher EDSS compared to NMOSD patients $(p=0.0091)$ (Table 1$)$.

The MS patients had a higher lesion load and brain involvement including supra- and infra-tentorial involvement. Larger number $(60 \%)$ of the NMOSD patients had gadolinium enhancement on MRI suggestive of active lesions compared to MS patients (45\%). OCB positivity among MS patients was $42.1 \%$ and AQP4 positivity among NMOSD group was 90\% (Table 2).

Cognitive impairment was observed in equal proportion and in very large number of subjects. The primary domains involved in MS patients were executive function (95\%), IPS (75\%), and attention (70\%). Praxis, gnosis, and visuo-perceptual ability (0\%) were not affected. In NMOSD group, the predominant domains involved were executive function (80\%) and IPS (70\%). Except for executive function which was more frequently affected in MS, the rest of the cognitive involvement did not yield any statistically significant difference between MS and NMOSD (Table 3).

Most affected executive subdomains involved in MS patients were mental flexibility (75\%), set shifting (70\%), and abstraction (55\%) and verbal fluency (30\%). The predominant executive subdomain involved in NMOSD patients was verbal fluency (80\%), mental flexibility (70\%), abstraction (60\%), and set shifting (50\%). The cognitive dysfunction profile between MS and NMOSD patients was found to be mostly similar except verbal fluency, 
Table 1 General characteristics of NMOSD and MS patients

\begin{tabular}{llll}
\hline Characteristics & MS $(\boldsymbol{n}=\mathbf{4 0})$ & NMOSD $(\mathbf{n}=\mathbf{2 0})$ & $\boldsymbol{P}$ value \\
\hline Age $($ mean \pm SD) & $34 \pm 7.34$ & $28.3 \pm 11.21$ & 0.0953 \\
Female:male & $1.5: 1$ & $2.3: 1$ & 0.5197 \\
Formal education in years (mean \pm SD) & $15.52 \pm 5.32$ & $11.40 \pm 13.31$ & 0.0057 \\
Duration in years (mean \pm SD) & $7 \pm .14$ & $2.12 \pm 2.22$ & $<0.0001^{*}$ \\
No. of relapses (mean \pm SD) & $2.75 \pm 1.06$ & $1.5 \pm 1.68$ & $<0.0001^{*}$ \\
EDSS & $3.23 \pm 1.94$ & $2.65 \pm 2.43$ & $0.0091^{*}$ \\
\hline
\end{tabular}

${ }^{*} p$ value $\leq 0.05$ is considered significant

and visuo-perceptual ability was involved more in NMOSD patients. No statistically significant difference was noted in the executive profile between the two groups except for verbal fluency and judgement, while verbal fluency dysfunction was noted more frequently in NMOSD, and impairment in judgement was noted more in MS (Table 4).

It was found that in MS patients, MRI lesion load (negative), and OCB positivity had statistically significant correlation with FAB scores. Moreover, age (positive), formal education (negative), and OCB positivity had statistically significant association with number of cognitive domain involvement. Similarly, formal education and OCB positivity had significant association with number of executive subdomain involvement. Thus, in MS patients, CI was more commonly prevalent in older patients with lesser formal education and larger MRI lesion load and OCB positivity.
In NMOSD patients, the FAB score and number of cognitive domains involved have statistically significant association with female gender and EDSS; whereas number of executive subdomains did not have any statistically significant association with other characteristics. Due to inadequate patients in the AQP4 negative arm, correlation of cognitive decline with AQP4 positivity could not be obtained. Thus, NMOSD patients who are females having higher disability scores seem to have higher cognitive dysfunction (Table 5).

\section{Discussion}

In our study, we found that MS patients were mostly middle aged with female preponderance, having higher formal educational status, longer duration of disease, and mild disability. MRI showed a higher lesion load with predominant supra-tentorial burden, highest being in the periventricular location. Cognitive impairment was present in a significant proportion of patients;

Table 2 MRI characteristics of NMOSD and MS patients

\begin{tabular}{|c|c|c|c|}
\hline Characteristics & MS $(n=40)$ & NMOSD $(n=20)$ & $P$ value \\
\hline \multicolumn{4}{|l|}{ MRI findings } \\
\hline No. of lesions & & & $<0.0001^{*}$ \\
\hline$\leq 4$ & $2(5 \%)$ & $16(80 \%)$ & \\
\hline $4-9$ & $6(15 \%)$ & $4(20 \%)$ & \\
\hline$\geq 9$ & $32(80 \%)$ & 0 & \\
\hline \multicolumn{4}{|l|}{ Lesion location } \\
\hline Periventricular & $40(100 \%)$ & $4(20 \%)$ & $<0.0001^{*}$ \\
\hline Juxta cortical & $22(55 \%)$ & $4(20 \%)$ & $0.0283^{*}$ \\
\hline Corpus callosal & $28(70 \%)$ & $2(10 \%)$ & $<0.0001^{*}$ \\
\hline Brain stem & $18(45 \%)$ & 0 & $0.0008^{*}$ \\
\hline Cerebellum & $10(25 \%)$ & 0 & $0.0096^{*}$ \\
\hline Area postrema & 0 & 0 & \\
\hline Diencephalon & 0 & 0 & \\
\hline Gadolinium enhancement & $18(45 \%)$ & $12(60 \%)$ & 0.3138 \\
\hline OCB & $16(42.1 \%)$ & $2(10 \%)$ & $0.002^{*}$ \\
\hline Anti-aquaporin 4 & 0 & 18(90\%) & $<0.0001^{*}$ \\
\hline
\end{tabular}

${ }^{*} p$ value $\leq 0.05$ is considered significant 
Table 3 Cognitive performance of NMOSD vs MS patients

\begin{tabular}{llll}
\hline Characteristic & MS & NMOSD & $\boldsymbol{P}$ value \\
\hline FAB & $14.35 \pm .24$ & $14.7 \pm 2.56$ & 0.7397 \\
Cognitive impairment (CI) & $32(80 \%)$ & $15(75 \%)$ & 0.65 \\
Predominant domain involved & Executive & Executive/ IPS & \\
No. of domains involved & $3.85 \pm 1.85$ & $3.5 \pm 2.21$ & 0.6579 \\
Attention & $28(70 \%)$ & $10(50 \%)$ & 0.3073 \\
IPS & $30(75 \%)$ & $14(70 \%)$ & 0.2563 \\
Language & $8(20 \%)$ & $2(10 \%)$ & 0.1793 \\
Memory & $10(25 \%)$ & $8(40 \%)$ & 0.2070 \\
Calculation & $22(55 \%)$ & $10(50 \%)$ & 0.0630 \\
Visuo-construction & $18(47.4 \%)$ & $10(62.5 \%)$ & 0.5929 \\
Visuo-perceptual & 0 & $2(11.1 \%)$ & 0.0572 \\
Praxis & 0 & 0 & \\
Gnosis & 0 & 0 & $0.006^{*}$ \\
Executive & $38(95 \%)$ & $14(70 \%)$ &
\end{tabular}

${ }^{*} p$ value $\leq 0.05$ is considered significant

No statistically significant difference was noted in cognitive performance between the two groups except for executive dysfunction

executive function (mental flexibility), IPS, and complex attention were maximally affected.

The NMOSD patients in our study had a younger age, female majority, lower formal educational status, shorter duration of disease, less frequent relapses, and mild disability. MRI indicated lower lesion load but more active lesions. Cognitive impairment was present in a noticeable number of patients with predominant affection of executive function (verbal fluency, IPS, visuo-construction, attention, and calculation).

The trends of cognitive involvement in our study of MS patients were similar to previous studies by Rao et al. [4], Amato et al. [16], Rima et al. [17], and the COGIMUS group [18], but the percentage of involvement noted in the present study was quite high. This

Table 4 Executive profile of NMOSD vs MS patients

\begin{tabular}{llll}
\hline Characteristic & MS & NMOSD & $\boldsymbol{P}$ value \\
\hline Predominant subdomain & $\begin{array}{l}\text { Mental } \\
\text { flexibility }\end{array}$ & $\begin{array}{l}\text { Verbal } \\
\text { fluency }\end{array}$ & \\
$\begin{array}{l}\text { No. of subdomains } \\
\text { involved }\end{array}$ & $2.5 \pm 1.13$ & $2.9 \pm 1.74$ & 0.25 \\
Abstraction & $22(55 \%)$ & $12(60 \%)$ & 0.8543 \\
Mental flexibility & $30(75 \%)$ & $14(70 \%)$ & 0.7008 \\
Verbal fluency & $12(30 \%)$ & $16(80 \%)$ & $<$ \\
Judgement & $2(5 \%)$ & 0 & $0.0001^{*}$ \\
Set shifting & $28(70 \%)$ & $10(50 \%)$ & $0.04392^{*}$ \\
Planning & $8(21.1 \%)$ & $6(37.5 \%)$ & 0.3470 \\
\hline
\end{tabular}

${ }^{*} p$ value $\leq 0.05$ is considered significant

No statistically significant difference was noted in the executive profile between the two group except for verbal fluency and judgement may be because of comprehensive assessment of patients and identification of even subtle cognitive impairment in various domains in the present study.

The educational status of the present study comprised of a mean of 15.52 years of formal education, graduates being the predominant group of patients. In the other studies, the years of formal education included 12.4 years in COGIMUS study [18], 11.44 in Amato et al. [16], and 15.9 years in Kaur et al. [19] study, while graduates were the predominant group in Rima et al. [17] group.

The mean duration from onset was 7 years and mean EDSS was 3.23 as compared to 14.2 years and 4.1 in Rao et al.'s study [4], 1.58 years and 1.98 in Amato et al. [16], 5 years and 1.8 in COGIMUS study [18], and 8.47 in Kaur et al. [19] and EDSS, and 3.15 in Rima et al. [17] group.

Negative correlation was found between FAB, age, and number of lesions on MRI which was statistically significant, probably because with increasing age and disease duration, there is cumulative accumulation of more lesions causing more axonal degeneration and brain atrophy.

Disease duration did not correlate with cognitive decline in a statistically significant manner in our present study as opposed to previous studies by Rao et al. and Amato et al. $[4,16]$. Disease duration have been considered as a predominant factor in the development of cognitive impairment in CNS demyelinating disorders, but it may be influenced by other factors also. The plausible explanations would include the theory of cognitive reserve, severity of the disease itself, hemispheres involved in the disease process, locations of the lesions and impairment of the functions subserved by them, and involvement of white matter alone or associated grey matter affection. All the above-mentioned factors can affect the course of cognitive functions independent of the disease duration [20].

The duration of the disease is not predictive of whether a patient will have cognitive impairment. Although cognitive impairment tends to progress over time in MS, it can and does appear at any time during the course of the illness, even as the first symptom. Conversely, patients who have had MS for many years may be completely free of cognitive deficits. However, it is rare for cognitive changes to disappear completely once they have appeared.

Total lesion load correlated with cognitive impairment is similar to our study, but we could not find significant correlation between EDSS and cognitive impairment as with some previous studies like that of Rao et al. [4], probably because EDSS is more so a marker of physical disability, and the substrate for the same is different from that of cognitive function. 
Table 5 Association between demographic, clinical, and MRI characteristics and cognitive dysfunction in MS and NMOSD patients

\begin{tabular}{|c|c|c|c|c|c|c|c|c|c|c|c|c|}
\hline \multirow[t]{2}{*}{ Characteristic } & \multicolumn{2}{|l|}{$\begin{array}{l}\text { MS } \\
\text { (FAB) }\end{array}$} & \multicolumn{2}{|c|}{$\begin{array}{l}\text { NMOSD } \\
\text { (FAB) }\end{array}$} & \multicolumn{2}{|c|}{$\begin{array}{l}\text { MS (no. of cognitive } \\
\text { domains involved) }\end{array}$} & \multicolumn{2}{|c|}{$\begin{array}{l}\text { NMOSD (no. of } \\
\text { cognitive domains } \\
\text { involved) }\end{array}$} & \multicolumn{2}{|c|}{$\begin{array}{l}\text { MS (no. of executive } \\
\text { subdomains involved) }\end{array}$} & \multicolumn{2}{|c|}{$\begin{array}{l}\text { NMOSD (no. of } \\
\text { executive subdomains } \\
\text { involved) }\end{array}$} \\
\hline & $r$ & $p$ & $r$ & $p$ & $r$ & $p$ & $r$ & $p$ & $r$ & $p$ & $r$ & $p$ \\
\hline Age & -.315 & .048 & -.309 & .185 & .446 & .004 & .197 & .404 & .309 & .053 & .238 & .311 \\
\hline Gender (female) & NA & .339 & NA & .009 & NA & .068 & NA & .009 & NA & .086 & NA & .531 \\
\hline $\begin{array}{l}\text { Formal education } \\
\text { in years }\end{array}$ & .301 & .051 & .385 & .093 & -.358 & .023 & -.263 & .261 & -0.632 & $<.0001$ & -.283 & .227 \\
\hline Disease duration & -.021 & .898 & .284 & .224 & .158 & .332 & -.029 & .902 & .219 & .175 & .044 & .854 \\
\hline No. of relapses & .146 & .368 & .233 & .323 & -.125 & .443 & -.060 & .802 & -.279 & .175 & -.114 & .633 \\
\hline EDSS & .101 & .534 & -.704 & .001 & -.097 & .550 & .514 & .020 & -.134 & .410 & .240 & .308 \\
\hline MRI lesion load & -.386 & .014 & -.040 & .867 & .268 & .094 & .232 & .325 & .292 & .068 & .176 & .457 \\
\hline OCB positivity & NA & .012 & NA & NA & NA & $<.0001$ & NA & NA & NA & $<.0001$ & NA & NA \\
\hline
\end{tabular}

The associations were calculated by Pearson's correlation coefficient and chi square tests. $r$ value is correlation coefficient. $p$ value $\leq 0.05$ considered statistically significant

Physical disability is likewise not predictive of cognitive impairment, and cognitive changes can occur even when physical symptoms are absent or very mild. Moreover, patients with substantial physical disability may be free of cognitive changes. It does appear, however, that if a patient has had MS for a long time and is severely disabled physically, the probability of cognitive impairment is increased to some extent.

The OCB positivity had positive correlation with FAB, cognitive, and executive impairment in MS patients which was statistically significant. The reason for the same can be higher disease activity indicated by intrathecal immunoglobulin synthesis.

The cognitive profile of NMOSD patients was similar to the previous international studies by Blanc et al. (2008) [5], Blanc et al. (2012) [21], Saji et al. [6], and He et al. [22], although executive involvement was much more in our study. The presence of brain lesions in MRI was $50 \%$ which was more than previous studies probably implying some association between the same and cognitive dysfunction. Also, in this study, definition of cognitive dysfunction was not the same as the abovementioned studies since we took into account even subtle changes in the cognitive domains. Moreover, the ethnicity and educational status as well as age of presentation may have influenced the difference. In our study, the domains of verbal fluency and visuoperceptual ability were more impaired in NMOSD patients compared to MS patients, whereas executive dysfunction was the most commonly affected domain in both the groups unlike previous studies. The reason for the same could be the educational background as well as the detailed executive examination of all the subdomains in the present study so as to identify the subtle cognitive impairments, if any. Moreover, the overall cognitive profile of the patients was in fitting with the subcortical type of cognitive impairment as is expected in these demyelinating conditions of the brain. The major studies on comparative cognitive profiling of MS and NMOSD have been summarized in Table 6 [6, 7, 23-25].

There has been previous documentation that despite the MS patient being younger, they had more impairment in variable cognitive domains compared to NMOSD probably attributed to the left superior temporal gyrus volume loss. However, our study results differed to those previous findings. The probable explanation could be related to the fact that though MS ultimately leads to cortical atrophy which indicates grey matter involvement, but both MS and NMOSD are primarily disorder of white matter (which primarily builds the primer of cognitive network) and thus it is not unusual to have a similar type of cognitive impairments. Moreover, no particular pattern of cognitive impairment is expected in NMOSD. Owing to the diverse cerebral involvement in NMOSD, the varying degree of cognitive impairments may be noted ranging from mild to florid multidomain impairment and will depend on the hemispheric involvement and location of the lesion. Thus, it can serve as another potential explanation for no particular difference in pattern of cognitive impairment except executive dysfunction between NMOSD and MS [26]. The highlight of the study is that a majority of patients of demyelinating disorders have some cognitive dysfunction however subtle, which can be detected by comprehensive cognitive assessment. Since the predominant age group affected is that of the high functioning youth and adult population involved in cognitively challenging jobs, hence even subtle cognitive dysfunction in the domains of attention, information processing speed, and executive function, which were primarily affected in our study, can lead to decreased productivity and occupational disability of these 


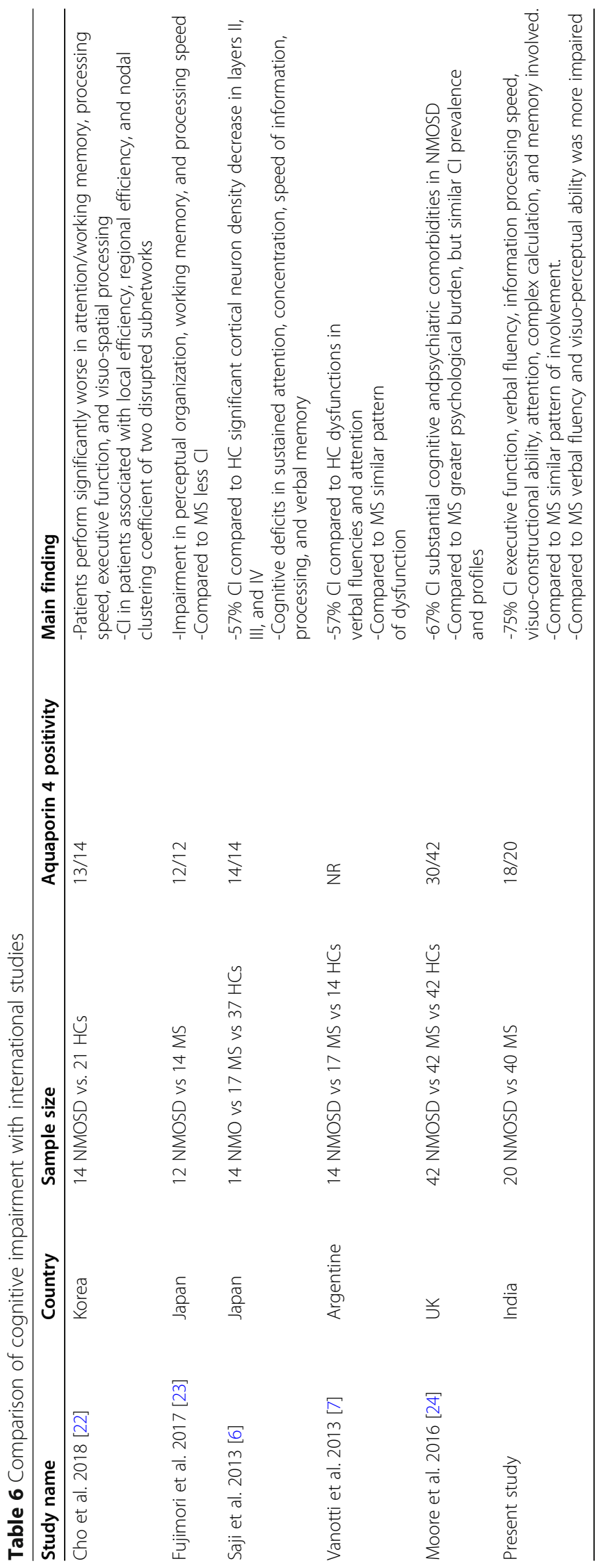


patients. A baseline and periodic assessment of cognitive function in these patients are therefore imperative for their professional well-being as well as timely intervention.

A smaller sample size without long-term follow-up and healthy controls, absence of recognized standard cognitive battery, and unavailability of newer MRI modalities to measure brain volume loss and functional deficit were the limitations of the study.

\section{Conclusions}

Cognitive impairment, although subtle, is present in significant number of patients with NMOSD and MS. The cognitive performances of MS and NMOSD patients are similar even though their clinical and radiological presentations are varied and different. Age at presentation, educational status, EDSS, MRI lesion load, and OCB positivity may influence the cognitive dysfunction in these patients. Standardized cognitive batteries, longterm follow-up studies and larger registries are needed for better understanding and management of this parameter.

\section{Abbreviations}

NMOSD: Neuromyelitis optica spectrum disorder; CNS: Central nervous system; AQP4: Aquaporin 4; Cl: Cognitive impairment; MS: Multiple sclerosis; IPND: International Panel for NMO Diagnosis; EDSS: Expanded Disability Status Scale; OCB: Oligoclonal band; FAB: Frontal assessment battery; KCB: Kolkata cognitive battery; MOCA: Montreal cognitive assessment; IPS: Information processing speed; ACE: Addenbrooke's cognitive examination; MMSE: Mini-Mental State Exam; MRI: Magnetic resonance imaging; FLAIR: Fluid attenuated inversion recovery; DWI: Diffusion-weighted imaging; ADC: Apparent diffusion coefficient; STIR: Short TI inversion recovery; GRE: Gradient echo; ANOVA: Analysis of variance;

COGIMUS: Cognitive impairment in multiple sclerosis

\section{Acknowledgements}

None.

\section{Authors' contributions}

SK, GG, and SDu were involved in patient care, data entry, and maintaining the demyelination registry. The initial concept and design of the study was generated by SK, GG, AB, and SDu. Literature search was done by SK and SDu. SK wrote the first draft which was subsequently edited by SDa and SDu. The statistical analysis was carried out by SK and SDu. The draft was critically reviewed by $S D u, G G$, and $A B$ from its initial stage. Expert opinion from AP and BR improved the quality of the final manuscript substantially. All the authors read and agreed upon the final form of the manuscript before submission.

\section{Funding}

No funding was received to assist with the preparation of this manuscript.

\section{Availability of data and materials}

All authors confirm the availability of data and materials.

\section{Declarations}

\section{Ethics approval and consent to participate}

Approval was obtained from the ethics committee of Institute of Postgraduate Medical Education \& Research, Kolkata, India. The procedures used in this study adhere to the tenets of the Declaration of Helsinki (Ethics Committee reference number ECR/35/Inst/WB/2013/RR-16). Written informed consent was obtained from all individual participants included in the study.

\section{Consent for publication}

Patients signed informed consent regarding publishing their data and photographs.

\section{Competing interests}

All authors certify that they have no affiliations with or involvement in any organization or entity with any financial interest or non-financial interest in the subject matter or materials discussed in this manuscript.

Received: 22 March 2021 Accepted: 4 July 2021

Published online: 21 July 2021

\section{References}

1. Kim HJ, Paul F, Lana-Peixoto MA, Tenembaum S, Asgari N, Palace J, et al. MRI characteristics of neuromyelitis optica spectrum disorder: an international update. Neurology. 2015;84(11):1165-73. https://doi.org/1 0.1212 /WNL.0000000000001367.

2. Kim SH, Kim W, Kook MC, Hong EK, Kim HJ. Central nervous system aquaporin-4 autoimmunity presenting with an isolated cerebra abnormality. Mult Scler. 2012;18(9):1340-3. https://doi.org/10.1177/13524 58512441271

3. Oertel FC, Schliesseit J, Brandt AU, Paul F. Cognitive impairment in neuromyelitis optica spectrum disorders: a review of clinical and neuroradiological features. Front Neurol. 2019;10:608. https://doi.org/10.33 89/fneur.2019.00608

4. Rao SM, Leo GJ, Bernardin L, Unverzagt F. Cognitive dysfunction in multiple sclerosis. I. Frequency, patterns, and prediction. Neurology. 1991;41(5):68591. https://doi.org/10.1212/wnl.41.5.685

5. Blanc F, Zephir H, Lebrun C, Labauge $P$, Castelnovo G, Fleury M, et al. Cognitive functions in neuromyelitis optica. Arch Neurol. 2008;65(1):84-8. https://doi.org/10.1001/archneurol.2007.16.

6. Saji E, Arakawa M, Yanagawa K, Toyoshima Y, Yokoseki A, Okamoto K, et al. Cognitive impairment and cortical degeneration in neuromyelitis optica. Ann Neurol. 2013;73(1):65-76. https://doi.org/10.1002/ana.23721.

7. Vanotti S, Cores EV, Eizaguirre B, Melamud L, Rey R, Villa A. Cognitive performance of neuromyelitis optica patients: comparison with multiple sclerosis. Arq Neuropsiquiatr. 2013;71(6):357-61. https://doi.org/10.1590/ 0004-282X20130038.

8. Polman CH, Reingold SC, Banwell B, Clanet M, Cohen JA, Filippi M, et al. Diagnostic criteria for multiple sclerosis: 2010 revisions to the McDonald criteria. Ann Neurol. 2011;69(2):292-302. https://doi.org/10.1002/ana.22366.

9. Wingerchuk DM, Banwell B, Bennett $J$, Cabre P, Carroll W, Chitnis T, et al. International consensus diagnostic criteria for neuromyelitis optica spectrum disorders. Neurology. 2015;85(2):177-89. https://doi.org/10.1212/WNL. 0000000000001729

10. Kurtzke JF. Rating neurologic impairment in multiple sclerosis: an expanded disability status scale (EDSS). Neurology. 1983:33(11):1444-52. https://doi. org/10.1212/wnl.33.11.1444.

11. Dubois B, Slachevsky A, Litvan I, Pillon B. The FAB: a frontal assessment battery at bedside. Neurology. 2000;55(11):1621-6. https://doi.org/10.1212/ wnl.55.11.1621.

12. Shyamal Kumar DAS, Tapas Kumar BANERJEE, Chandra Shekhar MUKHERJEE, Paramita BOSE, Atanu BISWAS, Avijit HAZRA, et al. An urban communitybased study of cognitive function among non-demented elderly population in India. Neurology Asia. 2006;11:37-48

13. Nasreddine ZS, Phillips NA, Bedirian V. Charbonneau S, Whitehead V. Collin I et al. The Montreal Cognitive Assessment, MoCA: a brief screening tool for mild cognitive impairment. J Am Geriatr Soc. 2005;53(4):695-9. https://doi. org/10.1111/j.1532-5415.2005.53221.x.

14. Mathuranath PS, Nestor PJ, Berrios GE, Rakowicz W, Hodges JR. A brief cognitive test battery to differentiate Alzheimer's disease and frontotemporal dementia. Neurology. 2000;55(11):1613-20. https://doi.org/1 0.1212/01.wnl.0000434309.85312.19.

15. Benedict RHBS, Groninger D, Dobraski L, Shpritz M, Barnett. Revision of the brief visuospatial memory test: studies of normal performance, reliability, and validity. Psychol Assess. 1996;8(2):145-53. https://doi.org/10.1037/1040-3 590.8.2.145

16. Amato MP, Ponziani G, Siracusa G, Sorbi S. Cognitive dysfunction in earlyonset multiple sclerosis: a reappraisal after 10 years. Arch Neurol. 2001; 58(10):1602-6. https://doi.org/10.1001/archneur.58.10.1602. 
17. Rima R. A cohort study of cognitive impairment in patients of multiple sclerosis. J Multiple Sclerosis. 2015;3(01):161. https://www.iomcworld.org/a bstract/a-cohort-study-of-cognitive-impairment-in-patients-of-multiplesclerosis-46442.html.

18. Patti F, Amato MP, Trojano M, Bastianello S, Tola MR, Goretti B, et al. Cognitive impairment and its relation with disease measures in mildly disabled patients with relapsing-remitting multiple sclerosis: baseline results from the Cognitive Impairment in Multiple Sclerosis (COGIMUS) study. Mult Scler. 2009;15(7):779-88. https://doi.org/10.1177/1352458509105544.

19. Kaur D, Kumar G, Singh AK. Quick screening of cognitive function in Indian multiple sclerosis patients using Montreal cognitive assessment test-short version. Ann Indian Acad Neurol. 2013;16(4):585-9. https://doi.org/10.4103/ 0972-2327.120478.

20. Leclercq E, Cabaret M, Guilbert A, Jougleux C, Vermersch P, Moroni C. Influence de la durée d'évolution de la maladie et de l'âge sur les troubles cognitifs de patients âgés atteints d'une sclérose en plaques de forme rémittente (SEP-RR). Geriatr Psychol Neuropsychiatr Vieil [Internet]. 2014 Sep 1 [cited 2021 Jun 4];12(3):331-8. Available from: https://pubmed.ncbi.nlm. nih.gov/25245320/

21. Blanc F, Noblet V, Jung B, Rousseau F, Renard F, Bourre B, et al. White matter atrophy and cognitive dysfunctions in neuromyelitis optica. PLoS One. 2012;7(4):e33878. https://doi.org/10.1371/journal.pone.0033878.

22. He D, Chen $X$, Zhao D, Zhou H. Cognitive function, depression, fatigue, and activities of daily living in patients with neuromyelitis optica after acute relapse. Int J Neurosci. 2011;121(12):677-83. https://doi.org/10.3109/002074 54.2011.608456.

23. Cho EB, Han CE, Seo SW, Chin J, Shin JH, Cho HJ, et al. White Matter Network Disruption and Cognitive Dysfunction in Neuromyelitis Optica Spectrum Disorder. Front Neurol. 2018;9:1104. https://doi.org/10.3389/ fneur.2018.01104.

24. Fujimori J, Nakashima I, Baba T, Meguro Y, Ogawa R, Fujihara K. Cognitive impairment in neuromyelitis optica spectrum disorders: A comparison of the Wechsler Adult Intelligence Scale-III and the Wechsler Memory Scale Revised with the Rao Brief Repeatable Neuropsychological Battery. eNeurologicalSci. 2017;9:3-7. https://doi.org/10.1016/j.ensci.2017.09.001.

25. Moore P, Methley A, Pollard C, Mutch K, Hamid S, Elsone L, et al. Cognitive and psychiatric comorbidities in neuromyelitis optica. J Neurol Sci. 2016;360: 4-9. https://doi.org/10.1016/j.jns.2015.11.031.

26. Masuda H, Hirano S, Takahashi N, Hatsugano E, Uzawa A, Uchida T, et al. Comparison of cognitive and brain grey matter volume profiles between multiple sclerosis and neuromyelitis optica spectrum disorder. PLoS One. 2017;12(8):e0184012. https://doi.org/10.1371/journal.pone.0184012 [cited 2021 Jun 4]

\section{Publisher's Note}

Springer Nature remains neutral with regard to jurisdictional claims in published maps and institutional affiliations.

\section{Submit your manuscript to a SpringerOpen ${ }^{\circ}$ journal and benefit from:}

- Convenient online submission

- Rigorous peer review

- Open access: articles freely available online

- High visibility within the field

- Retaining the copyright to your article

Submit your next manuscript at $\boldsymbol{\nabla}$ springeropen.com 